\title{
Severe infective endocarditis after MitraClip implantation treated by cardiac surgery
}

\author{
Christian Frerker ${ }^{*}$, MD; Karl-Heinz Kuck ${ }^{1}$, MD; Tobias Schmidt ${ }^{1}$, MD; Felix Kreidel ${ }^{1}$, MD; Ralf Bader ${ }^{2}$, MD; \\ Michael Schmoeckel², MD; Stephan Geidel², MD \\ 1. Department of Cardiology, Asklepios Klinik St. Georg, Hamburg, Germany; 2. Department of Cardiac Surgery, Asklepios \\ Klinik St. Georg, Hamburg, Germany
}

This paper also includes accompanying supplementary data published online at: http://www.pcronline.com/eurointervention/86th_issue/64

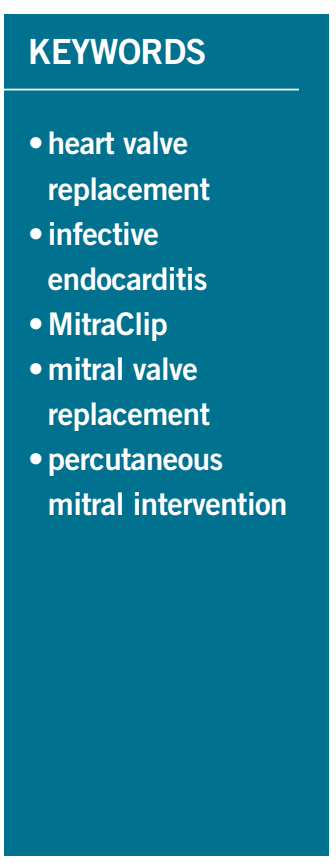

\begin{abstract}
Aims: To report the first described case of a mitral valve infective endocarditis (IE) post MitraClip ${ }^{\circledR}$.

Methods and results: An 88-year-old patient at high surgical risk (log. EuroSCORE 30.4\%) underwent a MitraClip procedure for severe eccentric organic mitral regurgitation (MR) due to prolapse with a flail leaflet of the P2 segment (flail gap $6 \mathrm{~mm}$ ). After one month, the patient was readmitted to our department for fever and recurrence of shortness of breath. An echocardiogram demonstrated severe MR recurrence due to ulceration in the region of the posterior leaflet despite good insertion of both clips, and a large vegetation of $15 \times 13 \mathrm{~mm}$ within the clip region. Blood cultures were positive for staphylococcus aureus. The patient was treated by cardiac surgery with mitral valve replacement due to IE despite a very high logistic EuroSCORE of $56.8 \%$. Histological and bacteriological analysis of the clip devices confirmed active IE. After cardiac surgery, transthoracic echocardiography showed no mitral regurgitation and a mean gradient across the mitral valve bioprosthesis of about $5 \mathrm{mmHg}$. The patient was discharged to a rehabilitation department 15 days after cardiac surgery.
\end{abstract}

Conclusions: Surgical treatment as bail-out therapy for MR recurrence secondary to IE after MitraClip can be successfully carried out despite a high surgical risk.

*Corresponding author: Department of Cardiology, Asklepios Klinik St. Georg, Lohmühlenstraße 5, 20099 Hamburg, Germany. E-mail: c.frerker@asklepios.com 


\section{Introduction}

The occurrence of mitral regurgitation (MR) is often associated with symptoms of heart failure as well as increased mortality $^{1}$. In the past, cardiac surgery was the standard therapy for these patients. With the introduction of the MitraClip ${ }^{\circledR}$ system (Abbott Vascular, Santa Clara, CA, USA) in 2008, there is now an alternative percutaneous treatment option available for high-risk patients. The EVEREST II trial and ACCESS EU registry demonstrated the safety and efficacy of the MitraClip ${ }^{2,3}$

We report on the first described case of a mitral valve infective endocarditis (IE) post MitraClip which was treated successfully by cardiac surgery.

\section{Case report}

An 88-year-old male patient was admitted to the emergency room with increasing shortness of breath at stage NYHA Class III. In the past, an atrial fibrillation and chronic kidney injury (CKI) stage III were known. On admission, medication consisted of aspirin, candesartan, metoprolol, and torasemide.

An echocardiogram showed severe eccentric organic MR due to prolapse with a flail leaflet of the P2 segment (flail gap $6 \mathrm{~mm}$ ), an enlarged left atrium of $60 \mathrm{~mm}$ and a normal ejection fraction of about $60 \%$ (Figure 1A, Figure 1B, Moving image 1 - Moving image 3). There were no signs of an infection: patient was afebrile with c-reactive protein and leukocytes at normal values. Despite initiation of diuretic medication for decompensated heart failure, there was only slight clinical improvement. Correction of the MR was discussed within our Heart Team. Due to age and comorbidities, such as pulmonary hypertension and CKI, a logistic (log) EuroSCORE of
$30.4 \%$ and a EuroSCORE II of $6.5 \%$ were calculated. As a result of high surgical risk, a MitraClip intervention was planned. The procedure was carried out in our hybrid operating room (OR) under aseptic conditions and laminar airflow. After implantation of two clips within the P2A2 segment, the MR was reduced to mild (Figure 1C, Figure 1D, Moving image 4). The gradient above the mitral valve was only $3 \mathrm{mmHg}$ at the end of the procedure. An antibiotic prophylaxis with intravenous cefazoline was given at the time of the procedure and for three more days according to our standard antibiotic regime. The patient was discharged from the hospital seven days after the intervention. Thirty-three days later the patient was readmitted to our department due to fever and recurrence of shortness of breath (NYHA Class III). Blood results showed elevated inflammatory parameters (CRP $163 \mathrm{mg} / \mathrm{L}$, leucocytes 16.8/nl). An echocardiogram demonstrated severe MR recurrence due to ulceration in the region of the posterior leaflet despite good insertion of both clips, and a large vegetation of $15 \times 13 \mathrm{~mm}$ within the clip region (Figure 2, Moving image 5, Moving image 6). The blood cultures were positive for staphylococcus aureus. According to the guidelines for IE, an antibiotic regime consisting of vancomycin, gentamicin and rifampicin was initiated. Due to the severe MR recurrence and the large vegetation we discussed the case again within our Heart Team. Despite his age and comorbidities a decision was taken, together with the patient, for cardiac surgery due to IE of the mitral valve.

At the time of surgery a $\log$ EuroSCORE of $56.8 \%$ and a EuroSCORE II of $25.3 \%$ were calculated. We performed the operation using a standard atriotomy, and antegrade Bretschneider cardioplegia was used for myocardial protection. Intraoperative valve analysis demonstrated severe IE of both mitral leaflets involving

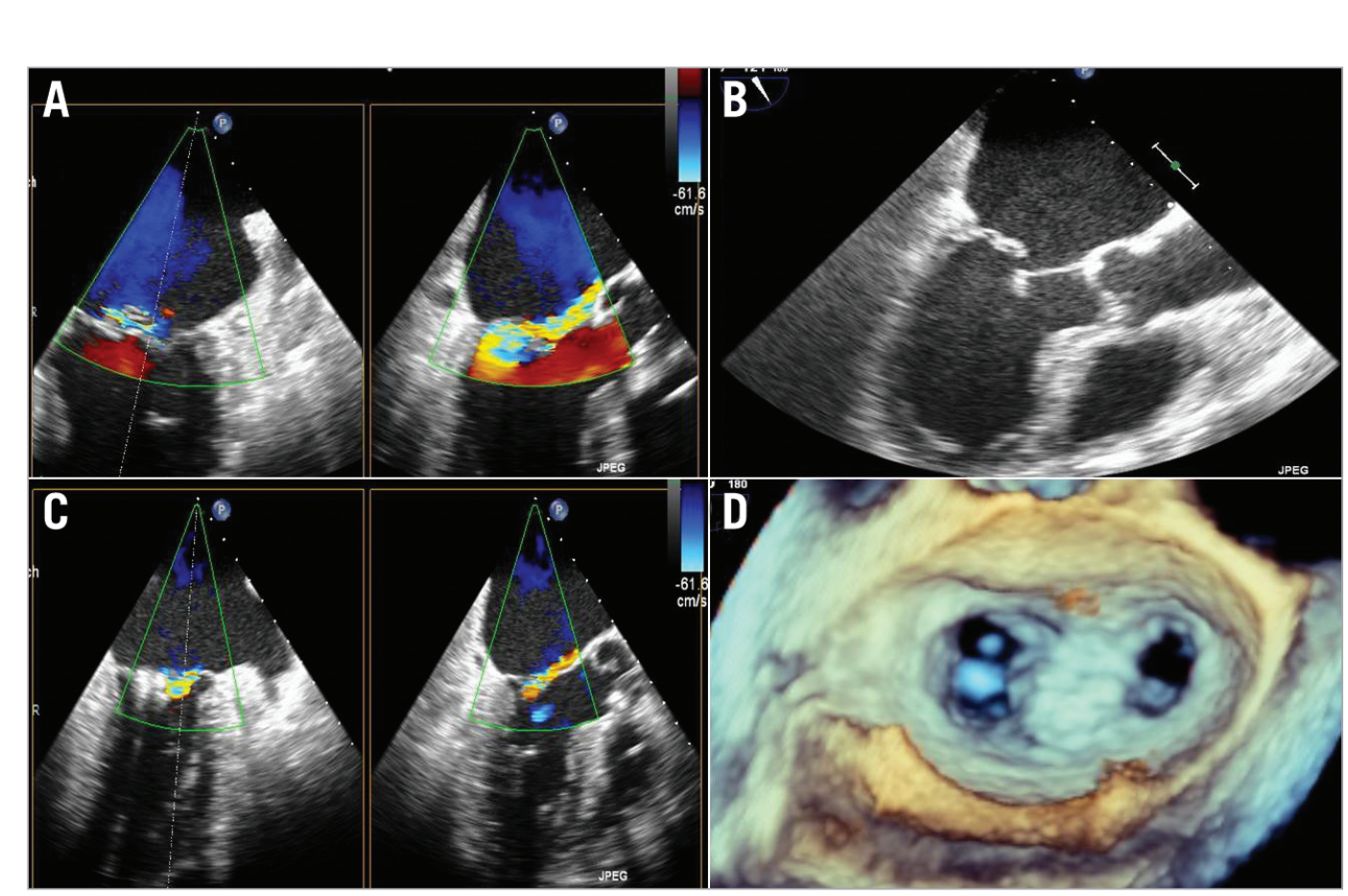

Figure 1. Transoesophageal echocardiography. A) Severe MR pre-procedure. B) Flail leaflet of the PML. C) Mild MR after implantation of two MitraClips. D) $3 D$ view of the mitral valve with two clips in place at the end of the procedure. 


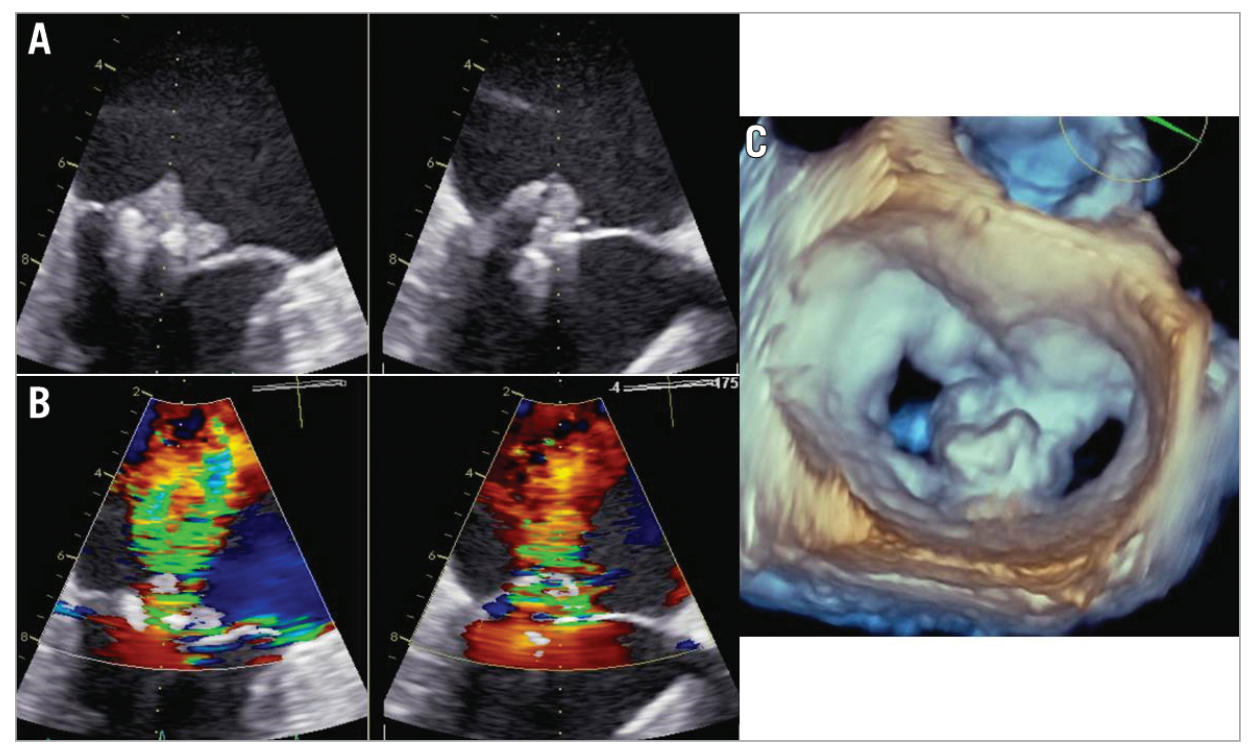

Figure 2. Transoesophageal echocardiography 33 days after MitraClip implantation. A) Large vegetation in the region of the clips. B) Severe central MR. C) $3 D$ view of the mitral valve with vegetation in the clip region.

the segments P1-3 and A1-3 (Figure 3). Mitral repair was therefore impossible. However, both clips had regularly gripped the anterior and the posterior leaflet in the segments A2/P2. Mitral valve resection was performed without difficulty, and a $33 \mathrm{~mm}$ bioprosthesis was implanted thereafter (Hancock ${ }^{\circledR}$ II; Medtronic, Minneapolis, MN, USA). Valve implantation was performed normally, as the mitral annulus was not involved in the infectious process. Closure of an artificial and a second congenital ASD, occlusion of the left atrial appendage and standardised radiofrequency ablation surgery were also performed. Aortic cross-clamping time and cardiopulmonary bypass time were 102 and $135 \mathrm{~min}$, respectively. Intraoperative transoesophageal echo demonstrated sufficient valve replacement without residual regurgitation. The patient was transferred to our intensive care unit and extubated on the first postoperative day. The postoperative course was uneventful. Histological and bacteriological analysis of the clip devices confirmed active IE.
After cardiac surgery, transthoracic echocardiography showed no mitral regurgitation and a mean gradient across the mitral valve bioprosthesis of about $5 \mathrm{mmHg}$. The patient was discharged 15 days after cardiac surgery to a rehabilitation department. Antibiotic medications were continued for an additional six weeks after surgery.

\section{Discussion}

The case illustrated here shows a surgical high-risk patient with severe organic MR who was treated by a MitraClip procedure. Various MitraClip ${ }^{\circledR}$ studies have demonstrated a safe and effective treatment option for surgical high-risk patients with severe $\mathrm{MR}^{2,3}$. The regular gripped leaflets, which were seen intraoperatively, confirm the initial successful reduction of MR by the MitraClip procedure. However, symptoms for IE must be taken seriously and antibiotic therapy is essential ${ }^{4}$. In this case, due to large vegetation, recurrent severe MR and symptoms of heart failure, a decision was taken within our Heart
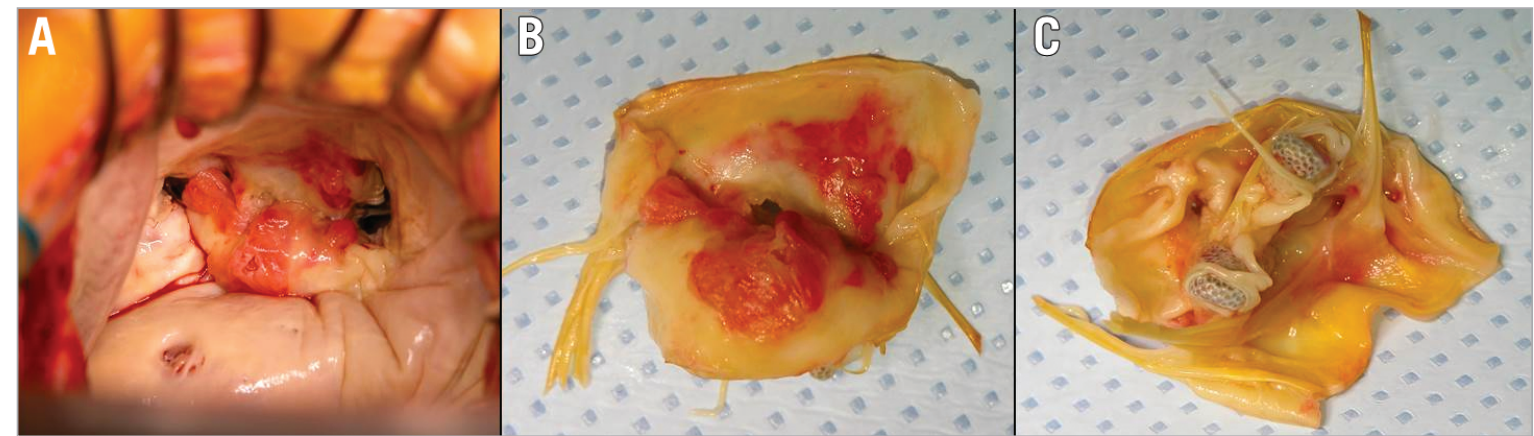

Figure 3. Intraoperative surgical view of the mitral valve. A) Exposure of the mitral valve after opening of the left atrium. Tissue shows severe IE involving both leaflets in the segments P1-3 and A1-3. B) Mitral valve after resection; ulcerations in segment P2 and the free edge of A2 are visible. The annulus is not involved in the infection. C) From the ventricular side the two ingrown MitraClips can be identified (segment P2); no chordal ruptures can be seen. 
Team for cardiac surgery. In accordance with studies which have demonstrated good results of cardiac surgery after failed mitral clipping, our patient also underwent an uneventful mitral valve replacement procedures. We believe that experience in a high-volume centre for MitraClip as well as for mitral valve surgery is important regarding good outcome in such surgical high-risk patients. Because of severe tissue infection of six mitral segments, valve repair was impossible in this case. This is the first described case of IE after a MitraClip implantation. In the literature, the incidence of surgical prosthetic valve endocarditis (PVE) has been described in up to $30 \%$ of all cases of infective endocarditis ${ }^{6}$. The leading cause of PVE is an infection with staphylococcus aureus as in our case. We believe that endocarditis prophylaxis for patients with MitraClip is therefore as important as it is for patients with surgical prosthetic valves.

In summary, this is the first reported case of IE after MitraClip implantation. Surgical treatment as bail-out therapy was successful despite a very high surgical risk situation.

\section{Impact on daily practice}

Treatment of mitral regurgitation with the MitraClip is a safe and successful procedure. Occurrence of an infective endocarditis after MitraClip as well as after mitral valve surgery is possible. Therefore, one should pay attention to prevent such an inflammatory situation. Nevertheless, cardiac surgery as bail-out strategy can be discussed in this special patient population group despite very high surgical risk. The presence of a Heart Team consisting of at least one cardiologist and one cardiac surgeon is recommended.

\section{Conflict of interest statement}

The authors have no conflicts of interest to declare.

\section{References}

1. Bursi F, Enriquez-Sarano M, Nkomo VT, Jacobsen SJ, Weston SA, Meverden RA, Roger VL. Heart failure and death after myocardial infarction in the community: the emerging role of mitral regurgitation. Circulation. 2005;111:295-301.

2. Mauri L, Foster E, Glower DD, Apruzzese P, Massaro JM, Herrmann HC, Hermiller J, Gray W, Wang A, Pedersen WR, Bajwa T, Lasala J, Low R, Grayburn P, Feldman T; EVEREST II Investigators. 4-year results of a randomized controlled trial of percutaneous repair versus surgery for mitral regurgitation. $J$ Am Coll Cardiol. 2013;62:317-28.

3. Maisano F, Franzen O, Baldus S, Schafer U, Hausleiter J, Butter C, Ussia GP, Sievert H, Richardt G, Widder JD, Moccetti T, Schillinger W. Percutaneous mitral valve interventions in the real world: early and 1-year results from the ACCESS-EU, a prospective, multicenter, nonrandomized postapproval study of the MitraClip therapy in Europe. $J$ Am Coll Cardiol. 2013;62:1052-61.

4. Habib G, Hoen B, Tornos P, Thuny F, Prendergast B, Vilacosta I, Moreillon P, de Jesus Antunes M, Thilen U, Lekakis J, Lengyel M, Muller L, Naber CK, Nihoyannopoulos P, Moritz A, Zamorano JL; ESC Committee for Practice Guidelines. Guidelines on the prevention, diagnosis, and treatment of infective endocarditis (new version 2009): the Task Force on the Prevention, Diagnosis, and Treatment of Infective Endocarditis of the European Society of Cardiology (ESC). Endorsed by the European Society of Clinical Microbiology and Infectious Diseases (ESCMID) and the International Society of Chemotherapy (ISC) for Infection and Cancer. Eur Heart J. 2009;30:2369-413.

5. Geidel S, Schmoeckel M. Impact of failed mitral clipping on subsequent mitral valye operations. Ann Thorac Surg. 2014;97: $56-63$.

6. Wang A, Athan E, Pappas PA, Fowler VG Jr, Olaison L, Pare C, Almirante B, Munoz P, Rizzi M, Naber C, Logar M, Tattevin P, Iarussi DL, Selton-Suty C, Jones SB, Casabe J, Morris A, Corey GR, Cabell CH; International Collaboration on EndocarditisProspective Cohort Study Investigators. Contemporary clinical profile and outcome of prosthetic valve endocarditis. JAMA. 2007;297:1354-61.

\section{Online data supplement}

Moving image 1. TEE split-screen intercommisural and LVOT view without colour. Flail leaflet PML.

Moving image 2. TEE LVOT view with colour. Flail leaflet PML. Moving image 3. 3D TEE view before MitraClip.

Moving image 4. TEE split-screen intercommisural and LVOT view with colour after implantation of two MitraClips.

Moving image 5. TEE split-screen intercommisural and LVOT view without colour and signs of endocarditis.

Moving image 6. TEE split-screen intercommisural and LVOT view with colour and signs of endocarditis. 